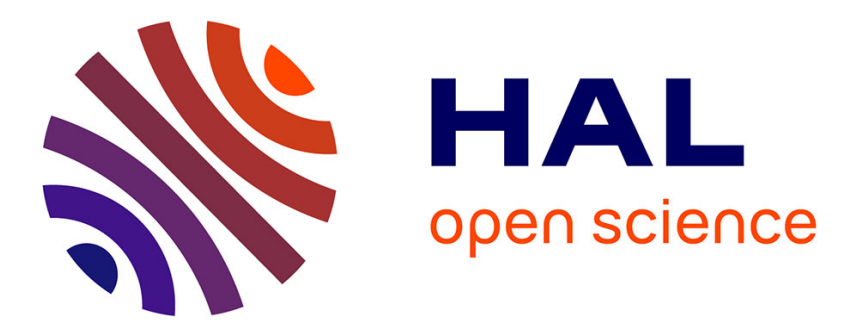

\title{
A Probabilistic Approach to Unsupervised Induction of Combinatory Categorial Grammar in Situated Human-Robot Interaction
}

\author{
Amir Aly, Tadahiro Taniguchi, Daichi Mochihashi
}

\section{- To cite this version:}

Amir Aly, Tadahiro Taniguchi, Daichi Mochihashi. A Probabilistic Approach to Unsupervised Induction of Combinatory Categorial Grammar in Situated Human-Robot Interaction. IEEE-RAS 18th International Conference on Humanoid Robots (Humanoids), Nov 2018, Beijing, China. hal-02004859

\section{HAL Id: hal-02004859 \\ https://hal.science/hal-02004859}

Submitted on 2 Feb 2019

HAL is a multi-disciplinary open access archive for the deposit and dissemination of scientific research documents, whether they are published or not. The documents may come from teaching and research institutions in France or abroad, or from public or private research centers.
L'archive ouverte pluridisciplinaire HAL, est destinée au dépôt et à la diffusion de documents scientifiques de niveau recherche, publiés ou non, émanant des établissements d'enseignement et de recherche français ou étrangers, des laboratoires publics ou privés. 


\title{
A Probabilistic Approach to Unsupervised Induction of Combinatory Categorial Grammar in Situated Human-Robot Interaction
}

\author{
Amir Aly ${ }^{1}$ and Tadahiro Taniguchi ${ }^{2}$ and Daichi Mochihashi ${ }^{3}$
}

\begin{abstract}
Robots are progressively moving into spaces that have been primarily shaped by human agency; they collaborate with human users in different tasks that require them to understand human language so as to behave appropriately in space. To this end, a stubborn challenge that we address in this paper is inferring the syntactic structure of language, which embraces grounding parts of speech (e.g., nouns, verbs, and prepositions) through visual perception, and induction of Combinatory Categorial Grammar (CCG) in situated humanrobot interaction. This could pave the way towards making a robot able to understand the syntactic relationships between words (i.e., understand phrases), and consequently the meaning of human instructions during interaction, which is a future scope of this current study.
\end{abstract}

\section{INTRODUCTION}

Creating interactive social robots able to collaborate with human users in different tasks requires high-level spatial intelligence that could make them able to discover and interact with their surroundings. Developing this spatial intelligence involves grounding language (action verbs, object characteristics (i.e., color and geometry), and spatial prepositions) and the underlying syntactic structure through sensory information so as to make a robot able to understand human instructions in the physical world.

Understanding syntactic structure of language has been intensively investigated in the literature of cognitive robotics and computational linguistics. In cognitive robotics, different research studies proposed computational models for grounding nouns, verbs, adjectives, and prepositions encoding spatial relationships between objects [1, 2, 24, 29, 42]. However, they have not investigated grammar understanding at the phrase level, which constitutes a higher level than grounding words through perception. Meanwhile, in computational linguistics, recent studies presented models for inducing combinatory syntactic structure of language $[5,16]$; however, they used annotated databases for grammar induction where each word has a corresponding syntactic tag (as a noun, verb, etc.). This last point illustrates the important role that cognitive robotics could play in grammar induction through grounding parts of speech in visual perception so as to allow for learning the latent syntactic structure of phrases in a developmentally plausible manner. In this study, we build on the model of Bisk and Hockenmaier [5] for grammar induction, and propose an extended probabilistic

\footnotetext{
${ }^{1}$ Amir Aly is with the Emergent Systems Laboratory, Ritsumeikan University, Japan amir.aly@em.ci.ritsumei.ac.jp.

${ }^{2}$ Tadahiro Taniguchi is with the Emergent Systems Laboratory, Ritsumeikan University, Japan taniguchi@em.ci.ritsumei.ac.jp.

${ }^{3}$ Daichi Mochihashi is with the Institute of Statistical Mathematics, Japan daichi@ism.ac.jp.
}

Bayesian framework for grounding parts of speech within a cross-situational learning context between a human user and a robot $[1,36]$. The overall structure of the system is coordinated through the following two phases:

1) Unsupervised calculation of Part-of-Speech (POS) tags for words (Section V), then grounding both words and tags through visual perception so as to learn their syntactic categories and meanings (Section VI) ${ }^{1}$.

2) Unsupervised induction of Combinatory Categorial Grammar (CCG) categories based on the grounded tags in phase (1) (Section VIII).

The results show that the proposed probabilistic framework for grounding parts of speech was able to successfully provide correct tags to the grammar induction model. This paves the way towards grounding phrases and their induced CCG complex categories so as to allow a robot to understand phrases (not only words) composing sentences, which constitutes a direction of future research.

\section{RELATED WORK}

Grounding language in perception is an important challenge in artificial intelligence, cognitive robotics, and natural language processing. The "Symbol Grounding" problem was defined by Harnad [19], which discusses assigning a meaning to a meaningless symbol (e.g., new word) through interaction with the surroundings. Tanenhaus et al. [40] investigated the effect of visual cues on language understanding. Roy et al. [31] introduced an architecture to provide perceptual and affordance representations of words. Matuszek et al. [25] introduced a probabilistic framework that employs categorial grammar to develop compositional representations of language and objects in the environment. Tellex et al. [42] and Dawson et al. [12] proposed probabilistic frameworks for grounding verbs and prepositions in utterances that encode spatial relationships between referents and landmarks. Siskind [35] developed a model for grounding semantics of verbs in short image sequences. Marocco et al. [24] proposed a framework for grounding action words through sensorimotor interaction with the environment. These interesting studies, inter alia, have not discussed inferring grammatical structure of phrases in a developmentally plausible way, which constituted our motivation for the proposed study. This could open the door to make a robot understand phrases,

\footnotetext{
${ }^{1}$ For example, the following instruction could be tagged as follows: (Raise, 1) (the, 5) (Red, 2) (Bottle, 4) (Near, 6) (the, 5) (Box, 4), where these ungrounded numerical tags represent the syntactic categories of words (i.e., Verb, Determiner, Adjective, Preposition, and Noun).
} 
through an unsupervised approach, which is a future research line of this study.

The literature of natural language processing reveals different approaches towards inferring syntactic structure of language from Part-of-Speech (POS) tagging to grammar induction. Church [11], Brill [7], and Goldwater and Griffiths [18], inter alia, discussed different approaches - supervised, semi-supervised, and unsupervised - for tagging a word sequence with syntactic attributes. On the way towards studying a deeper syntactic structure of language, Klein and Manning [22] proposed a generative model for learning constituency and dependency in language for unsupervised grammar induction using induced Part-of-Speech tags. Dependency parses are determined through unlabeled edges between constituents without any defined syntactic categories, but they can not detect non-local structures efficiently. Steedman [37] introduced the rich and universal lexicalized formalism: Combinatory Categorial Grammar (CCG), where each constituent is associated with a structured syntactic category that determines its relationship to adjacent constituents in a sentence. Besides, the CCG formalism could effectively interface the syntactic to semantic structures of language [38] so as to allow a robot to better interpret human instructions.

Different approaches to unsupervised grammar induction have been investigated in the literature $[5,6,16]$; however, these approaches disregarded learning lexical information of words and used annotated corpora. In this paper, we bridge between artificial intelligence, cognitive robotics, and natural language processing, and propose a framework for grounding lexical information of words through visual perception so as to infer the combinatorial syntactic structure of language in an unsupervised manner - within a situated human-robot interaction context. This could pave the way to investigate grounding phrases and their induced CCG categories through visual perception so as to understand the syntactic structure of phrases composing sentences, which is a future research line of this current study that was not sufficiently addressed in the related literature.

The rest of the paper is organized as follows: Section (III) describes the system architecture, Section (IV) illustrates the visual perceptual system, Sections (V and VI) describe the lexical tagging of words and the proposed grounding model, Section (VII) presents the experimental setup, Section (VIII) introduces the CCG syntactic formalism of language, Section (IX) discusses the obtained results, and Section (X) concludes the paper.

\section{SYSTEM ARCHITECTURE}

The proposed framework in this study is coordinated through: (1) System for visual perception: which outputs position coordinates of the human arm joints while manipulating objects, in addition to position coordinates of objects on a tabletop and their color and geometrical characteristics (Section IV), (2) Systems for syntactic structural representation of language: which represents language through syntactic tags and combinatorial categories (Sections V and VIII), and (3) Probabilistic generative model: which grounds words and their syntactic tags through visual perception (Section VI). The following sections in the paper discuss the proposed approach in detail.

\section{VISUAL PERCEPTUAL INFORMATION}

\section{A. Skeleton Tracking: Representation of Action Verbs}

The left-to-right HMM-based gesture model ${ }^{2}$ uses the tracked $(x, y, z)$ position coordinates ${ }^{3}$ of the human right-arm joints (Figure 1) (converted to the local coordinate system of the referent) as observations [1]. Five HMM models are used to represent five action verbs (Section VII). Each HMM model is trained ${ }^{4}$, during the cross-situational learning phase [36], on position coordinates of the arm joints while performing an action in different ways using the ExpectationMaximization (EM) algorithm [13]. The probabilities of evaluation of the test joint coordinates, through the different trained HMM models, are used to represent actions as observations in the probabilistic generative model (Section VI).

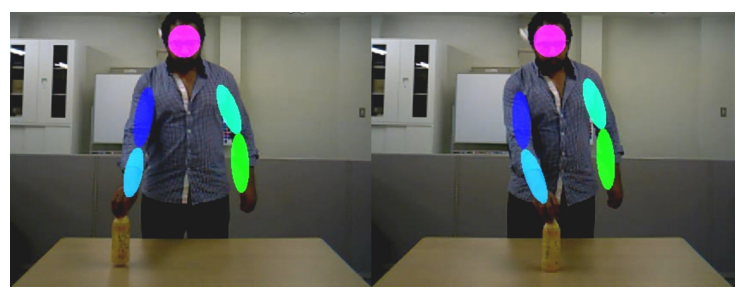

Fig. 1: Human body tracking and action characterization for object manipulation.

\section{B. Object Segmentation into Point Cloud: Representation of Spatial Concepts}

The unsupervised object segmentation model in the framework segments objects lying on a tabletop into distinct 3D point clouds with centroids representing their $(x, y, z)$ coordinates in respect of the robot camera (Figure 2) ${ }^{5}$. These coordinates allow the learning model to understand spatial concepts and relationships between objects. Each point cloud is characterized using its RGB color histogram and the Viewpoint Feature Histogram (VFH) descriptor [32] that could efficiently represent object geometry and viewpoint while being invariant to scale and pose. Having calculated object locations and features, the robot employs the probabilistic

\footnotetext{
${ }^{2}$ Hidden Markov Models (HMM) have been intensively used in the literature to model human body motion (a time-series observation sequence) in order to enable a robot to learn and generate behaviors without temporal constraints [20, 28, 39].

${ }^{3}$ The $3 \mathrm{D}$ tracking system uses the SDK OpenNI2 and the middleware NITE2.

${ }^{4}$ For each action verb in the training and test corpora (Section VII), position coordinates of the arm joints have been recorded during five different trials per action: three for training and two for testing.

${ }^{5}$ The model detects the tabletop plane using the RANSAC algorithm [14, 33], and the orthogonal wall planes in contact with, at least, one image border. The remaining points in the cloud are voxelized and clustered into distinct blobs representing object candidates. This model-based approach does not require prior knowledge about the environment such as neighboring information and the number of regions to process [21, 23].
} 
generative model (Section VI) in order to ground spatial concepts and object characteristics (i.e., color and geometry) through a cross-situational learning context with a human tutor in space [36].

\section{PART-OF-SPEECH TAGGING: UNGROUNDED LEXICAL TAGS OF WORDS}

The unsupervised ${ }^{6}$ Part-of-Speech (POS) tagging (tags induction) model assigns the numerical syntactic tag $\mathrm{T}=$ $\left(t_{1}, \ldots, t_{n}\right)$ to the word sequence $\mathrm{w}=\left(\omega_{1}, \ldots, \omega_{n}\right)$. The firstorder Hidden Markov Model (HMM) employs tags as hidden states and words as observations [15]. The probability distribution (transition) of the hidden tag states of the word sequence $\mathrm{w}$ is expressed as follows:

$$
\mathbb{P}\left(t_{1}, \ldots, t_{n}\right)=\prod_{i=1}^{n} \mathbb{P}\left(t_{i} \mid t_{i-1}\right)
$$

The emission distribution of tags over words is expressed through the probability $\mathbb{P}\left(\omega_{i} \mid t_{i}\right)$ of word $\omega_{i}$ conditioned on tag $t_{i}$. The emission and transition parameters $(\theta, \phi)$ are characterized using multinomial distributions with Dirichlet priors $\left(\alpha_{\theta}, \alpha_{\phi}\right)$ ( $K$ stands for the number of tag states):

$$
\begin{aligned}
\omega_{i} \mid t_{i} & =t \sim \operatorname{Mult}\left(\theta_{t}\right) \quad, \quad \theta_{t} \mid \alpha_{\theta} \sim \operatorname{Dir}\left(\alpha_{\theta}\right) \\
t_{i} \mid t_{i-1} & =t \sim \operatorname{Mult}\left(\phi_{t}\right), \quad \phi_{t} \mid \alpha_{\phi} \sim \operatorname{Dir}\left(\alpha_{\phi}\right)
\end{aligned}
$$

Having an unannotated corpus with a set of $m$ sentences $\mathrm{W}=\left\{w_{1}, \ldots, w_{m}\right\}$, the model assigns the most likely tag set $\mathrm{T}=\left\{T_{1}, \ldots, T_{m}\right\}$ - inferred using the Gibbs sampling algorithm [17] - to every sentence in the untagged corpus so as to maximize the following expression:

$$
\begin{array}{r}
\mathbb{P}(T, W)=\prod_{(T, w) \in(T, W)}(\mathbb{P}(T, w \mid \phi, \theta))= \\
\prod_{(T, w) \in(T, W)}\left(\prod_{i=1}^{n} \mathbb{P}\left(t_{i} \mid t_{i-1}, \phi_{t}\right) \mathbb{P}\left(\omega_{i} \mid t_{i}, \theta_{t}\right)\right)
\end{array}
$$

In addition to the HMM-based Part-of-Speech tagging model, we examine two other common unsupervised POS tagging models - using the same corpus - and we compare between their accuracies:

- BROWN Hierarchical Word Clustering: which assigns each word to a single cluster using an n-gram class conditional model [8].

- BMMM Clustering: which assigns words to clusters using a Bayesian Multinomial Mixture Model [10]. This model can incorporate different additional features on both the type and token levels of words leading to a precise calculation of POS tags.

Section (IX) provides measures for the accuracies of the three taggers, and the effect of each tagger on the word grounding and grammar induction processes.

\footnotetext{
${ }^{6}$ The literature reports different approaches to tagging parts of speech: (1) Supervised, which employs annotated training corpora to set up tagging dictionaries indicating possible tags to words [7], (2) Semi-supervised, which employs limited annotated corpora to estimate possible tags to new word sequences [43], (3) Unsupervised, which does not require any training corpus to assign tags to words [9].
}

\section{WORD GROUNDING IN PERCEPTION: A PROBABILISTIC GENERATIVE MODEL}

Figure (3) illustrates the multimodal Bayesian generative model used for grounding words (i.e., action verbs, spatial prepositions, and object characteristics) through visual perception with six observed states $\omega_{i}, z_{i}^{t}, a_{p}, c_{p}, s_{p}$, and $g_{p}$. The parameters of the generative model are defined in Table (I). The state $\omega_{i}$ stands for each word in the sequence $\mathrm{w}=$ $\left(\omega_{1}, \ldots, \omega_{n}\right)$, and the state $z_{i}^{t}$ stands for syntactic categories of words (Section V). The state $g_{p}$ stands for the geometrical characteristics of $O$ observed objects represented through the VFH descriptor (Section IV-B). The state $s_{p}$ stands for a spatial layout between a referent and a landmark represented through their centroid coordinates (Section IV-B). The state $c_{p}$ stands for the RGB color characteristics of $O$ observed objects (Section IV-B). The state $a_{p}$ stands for the arm joints locations while making actions on objects (Section IV-A). Having a spatial configuration between a referent and a landmark, the potential existing relationships between them could be expressed as follows: Observed Objects $O \times(O-1)$ (i.e., Referent A $\leftrightarrows$ Landmark B). The probabilistic distributions that characterize the Bayesian generative model are defined as follows (where GIW stands for a Gaussian InverseWishart distribution, Dir stands for a Dirichlet distribution, Cat stands for a categorical distribution, and Gauss stands for a multivariate Gaussian distribution) [3]:

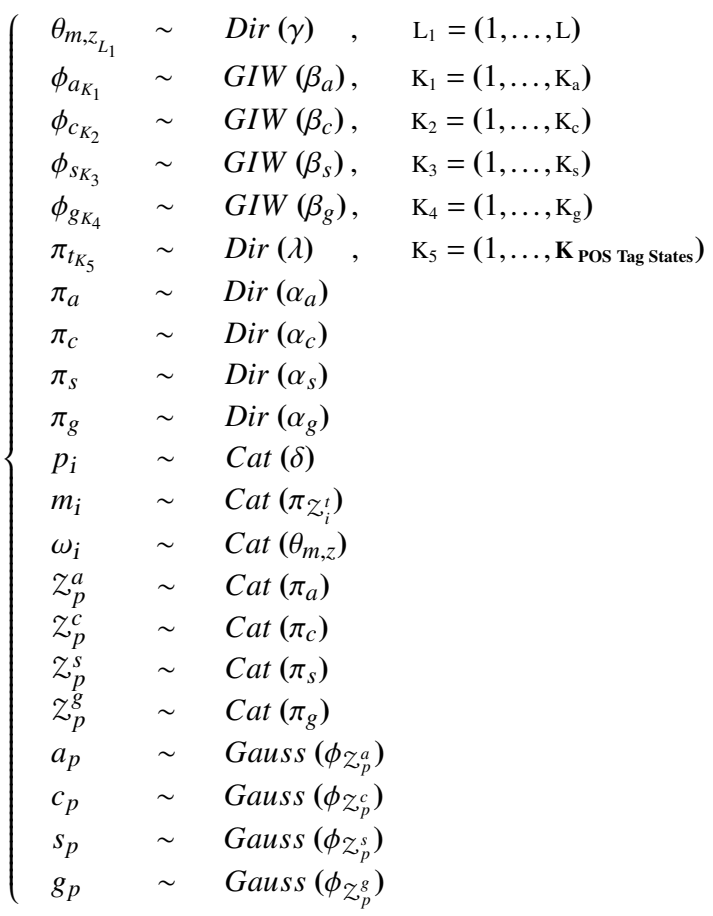

The latent variables are inferred using the Gibbs sampling algorithm [17] to allow the model to learn correspondences between words and their syntactic categories. The resulting grounded categories of words (illustrated with an example of a tagged human-to-robot instruction in footnote $(1)^{1}$, where those numerical tags are grounded through the generative model) are: Verb (representing action verbs), Adjective 


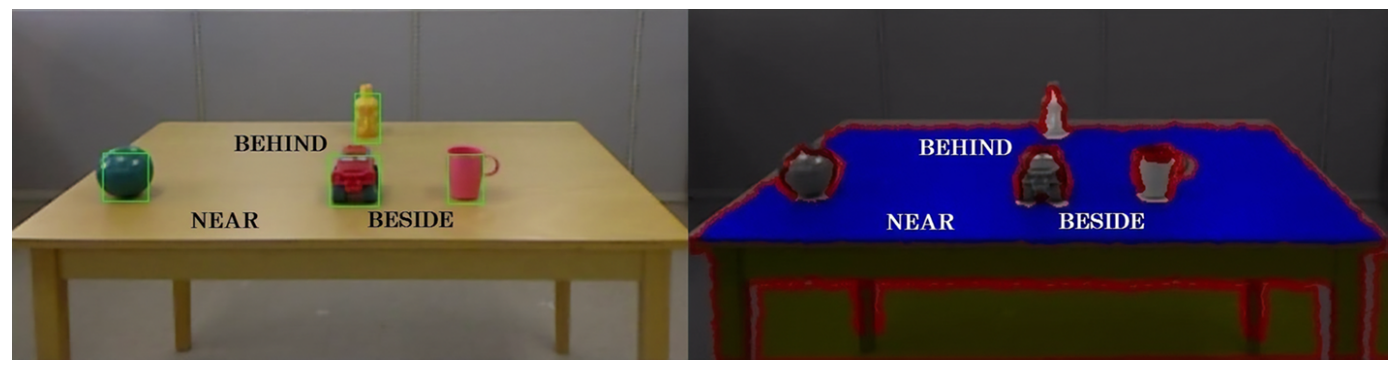

Fig. 2: Spatial concepts and relationships between objects represented through 3D point cloud information.

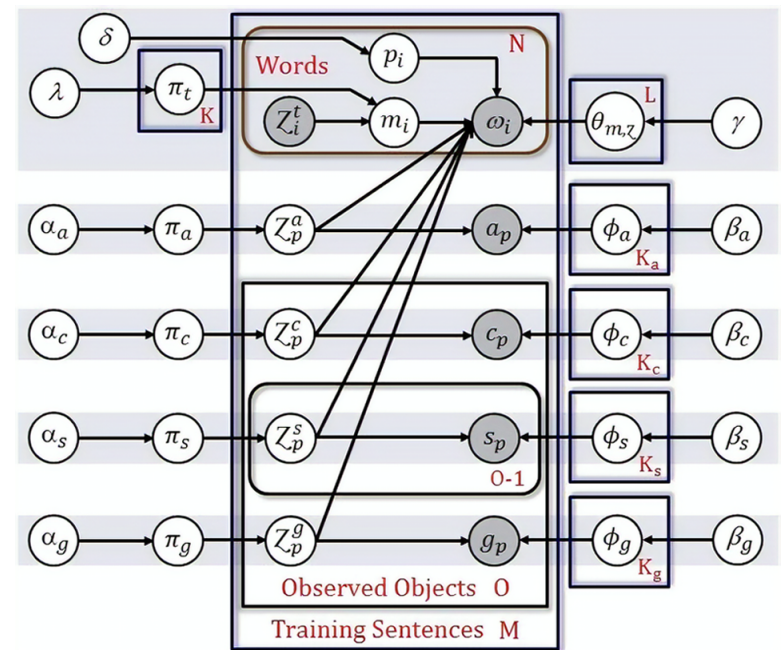

Fig. 3: Graphical representation of the probabilistic generative model. The order of words and POS tags is denoted by the index " $i$ ".

(representing object color), Preposition (representing spatial prepositions and relationships), Noun (representing object geometry: object name), and Determiner (representing an others category: the), which are used for grammar induction.

\section{EXPERIMENTAL SETUP}

A human tutor and the HSR robot $^{7}$ (Figure 4) are interacting in front of a table on which there are five different objects: \{Cup, Ball, Bottle, Toy, and $\mathrm{Box}^{8}$ \} with five different colors: \{Green, Yellow, Blue, Red, and White\} as referents and landmarks. In addition, we use five different prepositions: \{Above, Beside, Near, Behind, and Inside\} to represent spatial relationships between objects. Moreover, the robot executes five different actions: \{Put, RaIse, Hold, Pull, and Push (robot, object) ${ }^{9}$. The scenario of interaction

\footnotetext{
${ }^{7}$ The Human Support Robot (HSR) is developed by Toyota for providing assistance to people in daily life activities. It has a cylindrical shaped lightweight body with 11 degrees of freedom. The robot is equipped with one arm and a gripper to grasp objects, in addition to an array of sensors and cameras. [Toyota HSR Robot Website].

${ }^{8}$ The object 'Box' is considered only as a landmark.

${ }^{9} \mathrm{We}$ focus in this study on understanding actions on objects, therefore the action verbs were modeled on the robot that used the calculated distances to object centroids to control its joins and execute predefined behaviors. As a future extension of this study, we consider making the robot able to generate actions autonomously based on it learned experience [20].
}

TABLE I: Definitions of the model parameters in the different modalities.

\begin{tabular}{|c|c|}
\hline Parameter & Definition \\
\hline$\delta$ & Hyperparameter of the distribution $p_{i}$ \\
\hline$p_{i}$ & Index of spatial relationship (Object A $\leftrightarrows$ Object B) of each word \\
\hline$\lambda$ & Hyperparameter of the distribution $\pi_{t}$ \\
\hline$m_{i}$ & Index of word modality $\in$ \{Action, Color, Layout, Geometry, Others $\}$ \\
\hline$\gamma$ & Hyperparameter of the distribution $\theta_{m, z}$ \\
\hline$L$ & Number of word distribution categories $=K_{a}+K_{c}+K_{s}+K_{g}+1$ \\
\hline$\theta_{m, z}$ & Word distribution over modalities \\
\hline$\alpha_{a}$ & Hyperparameter of the distribution $\pi_{a}$ \\
\hline$\beta_{a}$ & Hyperparameter of the distribution $\phi_{a}$ \\
\hline$K_{a}$ & Number of categories in the action modality \\
\hline$\alpha_{c}$ & Hyperparameter of the distribution $\pi_{c}$ \\
\hline$\beta_{c}$ & Hyperparameter of the distribution $\phi_{c}$ \\
\hline$K_{c}$ & Number of categories in the object color modality \\
\hline$\alpha_{s}$ & Hyperparameter of the distribution $\pi_{s}$ \\
\hline$\beta_{s}$ & Hyperparameter of the distribution $\phi_{s}$ \\
\hline$K_{s}$ & Humber of categories in the spatial layout modality \\
\hline$\alpha_{g}$ & Hyperparameter of the distribution $\pi_{g}$ \\
\hline$\beta_{g}$ & Hyperparameter of the distribution $\phi_{g}$ \\
\hline$K_{g}$ & Number of categories in the object geometry modality \\
\hline$z_{p}^{a}$ & Index of action categories \\
\hline$z_{p}^{c}$ & Index of object color categories \\
\hline$z_{p}^{s}$ & Index of spatial layout categories \\
\hline$z_{p}^{g}$ & Index of object geometry categories \\
\hline
\end{tabular}

between the human tutor and the robot during the crosssituational learning phase [36] is summarized as follows:

- The tutor teaches the robot different spatial configurations of referents and landmarks lying in a tabletop described through 60 sentences - using visual perceptual information (Section IV). The unsupervised POS tagging model calculates numerical tags representing the syntactic categories of words for every training sentence (Section V).

- The visual information describing spatial layouts characterizes the dynamics of actions, object characteristics, and spatial relationships between objects with respect to the tabletop (Section IV).

- A probabilistic model grounds words through perception in order to define the necessary atomic categories for unsupervised CCG categories induction (Sections VI and VIII).

- The human tutor uses 30 test sentences describing different spatial layouts of objects (characterized through similar visual perceptual information as in the training phase and numerical POS tags) in order to validate the robustness of the word grounding process (Section IX). 


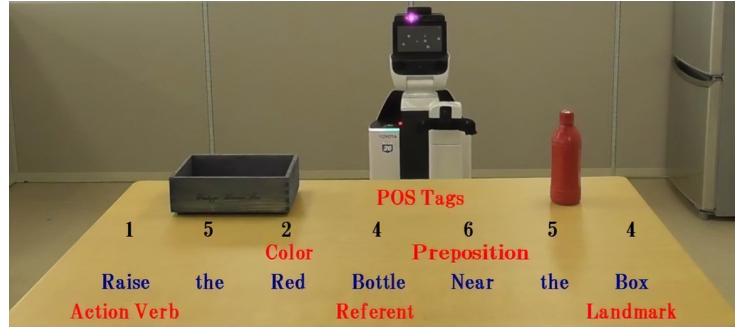

(a) In order to achieve the task, the robot needs to define the verb, the referent and its color, the landmark, and the existing spatial relationship between both objects through grounding words with their numerical POS tags in visual perception. The grounded POS tags are used for CCG categories induction.

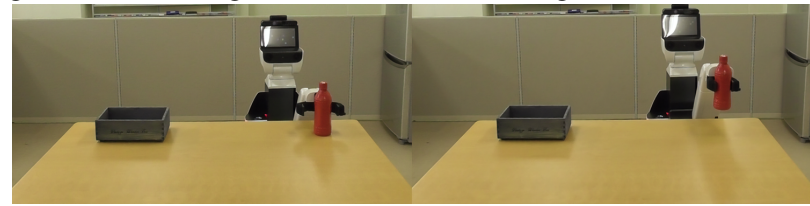

(b) The robot successfully raises the red bottle near the box.

Fig. 4: The robot achieves the assigned task through grounding words and POS tags in visual perception.

\section{COMBINATORY CATEGORIAL GRAMMAR: INFERRING SYNTACTIC STRUCTURE OF PHRASES}

Combinatory Categorial Grammar (CCG) is an expressive and a lexicalized syntactic formalism [37]. Any two syntactic categories amongst the atomic $(S, N$, and $N P$ ), functor (e.g., $N P / N$ ), or modifier (e.g., $N / N$ ) categories of neighboring constituents could be combined through a group of rules so as to create complex categories corresponding to higher level constituents. The slash operators: "/" indicates a forward combination (e.g., an argument follows a functor), and "\" indicates a backward combination (e.g., an argument precedes a functor). The standard unary and binary combinatory rules of the CCG formalism include [4]:

1) Application combinators:

$$
\overbrace{}^{\text {Functor }} \overbrace{}^{\text {Argument }}
$$

\begin{tabular}{|c|c|c|}
\hline - $\quad X / Y$ & $Y$ & $\stackrel{\text { Forward }>}{\longrightarrow}$ \\
\hline$Y$ & $X \backslash Y$ & \\
\hline$(X \backslash Z) / Y$ & $Y$ & $\stackrel{\text { Forward }>}{\Longrightarrow}$ \\
\hline$Y$ & $(X / Z) \backslash Y$ & $\stackrel{\text { Dackwara }}{\longrightarrow}$ \\
\hline
\end{tabular}

2) Composition combinators:

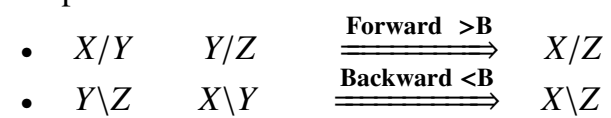

3) Type-raising unary combinators (argument $\Rightarrow$ functor):

$$
\begin{array}{llll}
\text { - } & Y & \text { Forward > T } & X /(X \backslash Y) \\
\text { - } & Y & \stackrel{\text { Backward <T }}{=} & X \backslash(X / Y)
\end{array}
$$

Figure (5) shows an example to illustrate the use of application combinators to create bottom-up parsing of constituents. The adjective "ReD" constitutes a modifier category

\begin{tabular}{|c|c|c|c|c|c|c|}
\hline \multirow{5}{*}{$\frac{\text { RAISE }}{S / N P}$} & THE & RED & BOTTLE & NEAR & THE & BOX \\
\hline & \multirow[t]{2}{*}{$\overline{N P / N}$} & $\overline{N / N}$ & $N$ & \multirow{2}{*}{$\overline{(N P \backslash N P) / N P}$} & $\overline{N P / N}$ & $N$ \\
\hline & & \multicolumn{2}{|r|}{$N$} & & $\mathrm{NI}$ & \\
\hline & \multicolumn{3}{|c|}{$N P$} & \multicolumn{3}{|c|}{$N P \backslash N P$} \\
\hline & & & & $N P$ & & \\
\hline
\end{tabular}
in the CCG parsing structure (i.e., it is assigned the category $N / N$ with both arguments before and after the slash operator
Fig. 5: CCG parsing through forward and backward application combinators (maximal arity equals 2 ).

are identical atomic categories). This allows some parts of speech to have generic categories that modify their head arguments (e.g., adjectives modify nouns, and adverbs modify verbs), unlike a functor category (e.g., $X / Y \rightarrow N P / N$ ) that represents a head category for its dependent argument.

Grounding each word and its induced POS tag (Sections VII and V) through visual perceptual information (Section IV) using the probabilistic generative model (Section VI) produces the categories: Verb, Determiner, Adjective, Preposition, and Noun. These syntactic categories define the atomic categories of the CCG formalism ${ }^{10}$. Having induced these atomic categories, the CCG induction model - proposed by Bisk and Hockenmaier [5] - learns the latent syntactic structure of sentences in the learning database, and generates combinatory syntactic categories for sentences in the test database (Section VII) so as to validate the robustness of the grammar induction process through comparison to a goldstandard parse structure (Section IX).

The Bayesian nonparametric HDP-CCG induction model employs Hierarchical Dirichlet Processes (HDP) [41] to generate an infinite set of CCG categories based on a stick-breaking model for each Dirichlet process [34] and multinomial distributions over arguments and combinators. The resulting induced CCG categories of constituent words and phrases could be combined together using the rules explained earlier that represent the combinatorial structure of phrases. Section (IX) provides measures for the accuracy of inducing CCG syntactic categories in different cases of tagging models.

\section{RESULTS AND DISCUSSION}

The framework is evaluated through its ability to induce correct CCG categories using the grounded POS tags. In this section, we provide evaluation for the accuracies of the different sub-models in the framework:

Part-of-Speech Tagging: Table (II) illustrates different measures for evaluating the robustness of the POS tagging process: V-Measure $^{11}$, VI-Measure ${ }^{12}$, and Many-to-One

\footnotetext{
${ }^{10}$ Noun Phrase $(\mathrm{NP})=$ Determiner + Noun $(\mathrm{N})$.

${ }^{11}$ It measures homogeneity (i.e., optimal case: each cluster (separate word category) contains fewer classes of tags) and completeness (i.e., optimal case: classes of tags referring to the same cluster are equal) of clusters and classes [30].

${ }^{12}$ It measures the variation of information of a clustering solution, so that the more the clustering is complete (i.e., high V-Measure), the lower the VI-Measure would be [26].
} 
(M-1)-Measure ${ }^{13}$. The difference in the V-Measure scores of the different POS taggers show that the HMM-based model was lower evaluated than the other two models.

TABLE II: Evaluation of unsupervised POS tagging through different measures.

\begin{tabular}{|c|c|c|c|}
\cline { 2 - 4 } \multicolumn{1}{c|}{} & M-1 Measure (\%) & V-Measure (\%) & VI-Measure \\
\hline BROWN Clustering & 100 & 94,9 & 0,24 \\
\hline HMM Clustering & 100 & 88,67 & 0,57 \\
\hline BMMM Clustering & 100 & 94,9 & 0,24 \\
\hline
\end{tabular}

Having a referent and a landmark in each sentence in the corpus, the HMM-based model assigned two different tags to all referents and landmarks in the corpus (i.e., all referents had a similar tag and all landmarks had another similar tag), which could clearly reduce the completeness score of the model (Table II). Meanwhile, the BMMM and BROWN models assigned the same tag to referents and landmarks, except for some sentences in the corpus where the tags of landmarks were assigned differently, which could reduce the completeness scores of both models as well. The effect of these tagging models on the word grounding and grammar induction processes will be discussed next.

Word Grounding: Grounding words through visual perception has the objective of defining word modality and spatial relationships between objects ${ }^{14}$. Tables (III) and (IV) illustrate the results of word grounding in case of the 3 POS tagging models, which show a similar performance of the three models when estimating word modality, and indicate some differences between them when determining correct spatial relationships between referents and landmarks.

TABLE III: Estimation of word modality using different tagging models.

\begin{tabular}{|c|c|c|c|c|}
\cline { 2 - 5 } \multicolumn{1}{c|}{} & \multicolumn{4}{c|}{ Correct Word Grounding (\%) } \\
\cline { 2 - 5 } \multicolumn{1}{c|}{} & Verb & Adjective & Preposition & Noun (Referent \& Landmark) \\
\hline BROWN Clustering & 76,7 & 100 & 66,7 & 60 \\
\hline HMM Clustering & 73,3 & 100 & 63,3 & 71,7 \\
\hline BMMM Clustering & 76,7 & 100 & 66,7 & 60 \\
\hline
\end{tabular}

TABLE IV: Correct referent-landmark spatial relationships represented through different prepositions.

\begin{tabular}{|c|c|c|c|c|c|}
\cline { 2 - 6 } \multicolumn{1}{c|}{} & \multicolumn{5}{c|}{ Correct Spatial Relationships Between Objects (\%) } \\
\cline { 2 - 6 } \multicolumn{1}{c|}{} & Above & Beside & Near & Behind & Inside \\
\hline BROWN Clustering & 0 & 83,3 & 100 & 33,3 & 100 \\
\hline HMM Clustering & 100 & 66,7 & 57,1 & 83,3 & 50 \\
\hline BMMM Clustering & 0 & 66,7 & 85,7 & 33,3 & 50 \\
\hline
\end{tabular}

The HMM-based tagging model achieved higher scores, in average, than the other two models in the grounding process (more specifically in Table IV with the preposition Aвоve), despite that it had a lower V-Measure score than the other models (Table II). This could be related to that the HMMbased model was able to better provide a unified tag to all landmarks, unlike the other models as explained earlier.

\footnotetext{
${ }^{13}$ Mapping between clusters and tags.

${ }^{14}$ Despite the rich literature in language grounding, we could not find a similar study in the approach, experimental setup, or corpus to the current one, which makes comparing these results to those of the other studies difficult to achieve.
}

However, these differences do not finally affect the accuracy of the grounding process in a global scope.

CCG Categories Induction: For the CCG induction process, we use the grounded parts of speech expressed through the standard tag set of the Penn Treebank Project ${ }^{15}$ : Verb: VB, Determiner: DT, Adjective: JJ, Preposition: IN, and Noun: NN as input to the CCG induction model, which learns the latent syntactic structure of sentences in the learning corpus so as to generate parse trees for sentences in the test corpus. These syntactic parses are highly dependent on the grounded tags, so that wrong tags could generate imprecise parse trees. To evaluate the robustness of the CCG induction process, we use a gold-standard parse file of all sentences in the test corpus to compare against. This file contains correct POS tags and dependency relations between words in each sentence that indicate edges of standard parse trees $^{16}$. We compare these edges to those resulting from the CCG model's predicted parses by calculating the number of matching edges.

TABLE V: Accuracy of CCG categories induction using different tagging models.

\begin{tabular}{|c|c|}
\cline { 2 - 2 } \multicolumn{1}{c|}{} & CCG Categories Induction / Matching Edges (\%) \\
\hline BROWN Clustering & 55,2 \\
\hline HMM Clustering & 59,4 \\
\hline BMMM Clustering & 60,6 \\
\hline Gold-POS (Without Grounding Model) & 68,2 \\
\hline
\end{tabular}

Table (V) illustrate the accuracy of CCG categories induction in case of the 3 POS tagging models in comparison to the case where the grounding model was not employed so that grammar induction was based only on gold-POS tags. This indicates that the BMMM and HMM-based tagging models showed, approximately, a similar good performance in grammar induction. These findings illustrate the ability of the framework to associate correct word grounding to grammar induction so as to investigate the combinatorial syntactic structure of language in an unsupervised manner. Similarly, they open the door to extend this framework to ground the generated CCG categories through perception in order to allow a robot to understand complex phrases during interaction. This includes understanding those phrases that encode spatial relationships expressed through prepositions composed of more than one word (e.g., in front of the Box), which would constitute a big step towards making robots able to collaborate effectively with human users in space.

\section{CONCLUSION AND FUTURE WORK}

This study presents a probabilistic framework for unsupervised induction of combinatory syntactic structure of language within a human-robot interaction context. The framework calculates numerical tags representing words in an unsupervised manner, and grounds them through visual perception so as to understand the syntactic categories and

\footnotetext{
${ }^{15}$ Penn Treebank Part-of-Speech Tag Set.

${ }^{16}$ These syntactic dependencies between words are calculated using Stanford Parser for evaluation only.
} 
meaning of words. These grounded words are used for inducing CCG categories, which builds on the current state-of-theart where a fully annotated corpus is used for grammar induction [5]. We discuss the effect of three POS tagging models on the word grounding and grammar induction processes, where the HMM-based tagging model showed a slightly better performance, in overall, than the other models. The evaluation scores of the generated CCG parses are promising and could be further improved through ameliorating the inference process of the HDP-CCG model, which we are considering to implement.

In our future work, we will consider representing the induced CCG categories of phrases in a compositional vector space - inspired by the research study of Nickel et al. [27] on holographic embeddings - with the objective of grounding phrases in perception, which would be a crucial step towards making robots able to understand language appropriately during interaction with human users.

\section{ACKNOWLEDGMENT}

This work was supported by AIP-PRISM, Japan Science and Technology Agency "JST". Grant number JPMJCR18Z4, Japan.

\section{REFERENCES}

[1] A. Aly and T. Taniguchi. Towards understanding objectdirected actions: A generative model for grounding syntactic categories of speech through visual perception. In Proceedings of the IEEE International Conference on Robotics and Automation (ICRA), Brisbane, Australia, 2018. 1, 2

[2] A. Aly, A. Taniguchi, and T. Taniguchi. A generative framework for multimodal learning of spatial concepts and object categories: An unsupervised part-of-speech tagging and 3D visual perception based approach. In Proceedings of the 7th Joint IEEE International Conference on Development and Learning and on Epigenetic Robotics (ICDL-EpiRob), Lisbon, Portugal, 2017. 1

[3] C. M. Bishop. Pattern Recognition and Machine Learning. Springer, 2006. 3

[4] Y. Bisk and J. Hockenmaier. Simple robust grammar induction with combinatory categorial grammars. In Proceedings of the Twenty-Sixth AAAI Conference on Artificial Intelligence, volume 2, pages 1643-1649, Toronto, Canada, 2012. 5

[5] Y. Bisk and J. Hockenmaier. An HDP model for inducing combinatory categorial grammars. Transactions of the Association for Computational Linguistics, 1:7588, 2013. 1, 2, 5, 7

[6] P. Boonkwan and M. Steedman. Grammar induction from text using small syntactic prototypes. In Proceedings of the 5th International Joint Conference on Natural Language Processing (NLP), pages 438-446, Chiang Mai, Thailand, 2011. 2

[7] E. Brill. A simple rule-based part of speech tagger. In Proceedings of the 3rd Conference on Applied Natural Language Processing (ANLC), Trento, Italy, 1992. 2, 3
[8] P. F. Brown, P. V. deSouza, R. L. Mercer, V. J. Della Pietra, and J. C. Lai. Class-based n-gram models of natural language. Computational Linguistics, 18(4): 467-479, 1992. 3

[9] C. Christodoulopoulos, S. Goldwater, and M. Steedman. Two decades of unsupervised POS induction: How far have we come? In Proceedings of the 15th Conference on Empirical Methods in Natural Language Processing (EMNLP), pages 575-584, Cambridge MA, USA, 2010. 3

[10] C. Christodoulopoulos, S. Goldwater, and M. Steedman. A Bayesian mixture model for Part-of-Speech induction using multiple features. In Proceedings of the Conference on Empirical Methods in Natural Language Processing (EMNLP), pages 638-647, Edinburgh, Scotland, 2011. 3

[11] K. W. Church. A stochastic parts program and noun phrase parser for unrestricted text. In Proceedings of the 2nd Conference on Applied Natural Language Processing (ANLC), Austin TX, USA, 1988. 2

[12] C. R. Dawson, J. Wright, A. Rebguns, M. V. Escarcega, D. Fried, and P. R. Cohen. A generative probabilistic framework for learning spatial language. In Proceedings of the 3rd Joint IEEE International Conference on Development and Learning and on Epigenetic Robotics (ICDL-EpiRob), Osaka, Japan, 2013. 1

[13] A. P. Dempster, N. M. Laird, and D. B. Rubin. Maximum likelihood from incomplete data via the EM algorithm. Journal of the Royal Statistical Society, Series B (Methodological), 39:1-38, 1977. 2

[14] M. A. Fischler and R. C. Bolles. Random Sample Consensus: A paradigm for model fitting with applications to image analysis and automated cartography. Communications of the ACM (CACM), 24(6):381-395, 1981. 2

[15] J. Gao and M. Johnson. A comparison of Bayesian estimators for unsupervised Hidden Markov Model POS taggers. In Proceedings of the 13th Conference on Empirical Methods in Natural Language Processing (EMNLP), pages 344-352, Honolulu HI, USA, 2008. 3

[16] D. Garrette, C. Dyer, J. Baldridge, and N. A. Smith. A supertag-context model for weakly-supervised CCG parser learning. In Proceedings of the 19th Conference on Computational Natural Language Learning (CoNLL), pages 22-31, Beijing, China, 2015. 1, 2

[17] S. Geman and D. Geman. Stochastic relaxation, Gibbs distributions and the Bayesian restoration of images. IEEE Transactions on Pattern Analysis and Machine Intelligence (TPAMI), 6(6):721-741, 1984. 3

[18] S. Goldwater and T. L. Griffiths. A fully Bayesian approach to unsupervised part-of-speech tagging. In Proceedings of the 45th Annual Meeting of the Association of Computational Linguistics (ACL), pages 744751, Prague, Czech Republic, 2007. 2

[19] S. Harnad. The symbol grounding problem. Physica D, 42:335-346, 1990. 1

[20] T. Inamura, I. Toshima, H. Tanie, and Y. Nakamura. 
Embodied symbol emergence based on mimesis theory. The International Journal of Robotics Research (IJRR), 23:363-377, 2004. 2, 4

[21] X. Y. Jiang, U. Meier, and H. Bunke. Fast range image segmentation using high-level segmentation primitives. In Proceedings of the 3rd IEEE International Workshop on Applications of Computer Vision (WACV), Sarasota Fl, USA, 1996. 2

[22] D. Klein and C. D. Manning. Corpus-based induction of syntactic structure: Models of dependency and constituency. In Proceedings of the 42nd Annual Meeting of the Association for Computational Linguistics (ACL), pages 478-485, Barcelona, Spain, 2004. 2

[23] K. Koster and M. Spann. MIR: An approach to robust clustering application to range image segmentation. IEEE Transaction on Pattern Analysis and Machine Intelligence (TPAMI), 22(5), 2000. 2

[24] D. Marocco, A. Cangelosi, K. Fischer, and T. Belpaeme. Grounding action words in the sensorimotor interaction with the world: Experiments with a simulated iCub humanoid robot. Frontiers in Neurorobotics, 4(7), 2010. 1

[25] C. Matuszek, N. FitzGerald, L. Zettlemoyer, L. Bo, and D. Fox. A joint model of language and perception for grounded attribute learning. In Proceedings of the 29th International Conference on Machine Learning (ICML), Edinburgh, Scotland, 2012. 1

[26] M. Meila. Comparing clusterings - an information based distance. Journal of Multivariate Analysis, 98 (5):873-895, 2007. 5

[27] M. Nickel, L. Rosasco, and T. Poggio. Holographic embeddings of knowledge graphs. In Proceedings of the Thirtieth AAAI Conference on Artificial Intelligence (AAAI), pages 1955-1961, Phoenix, Arizona USA, 2016. 7

[28] K. Ogawara, J. Takamatsu, H. Kimura, and K. Ikeuchi. Modeling manipulation interactions by Hidden Markov Models. In Proceedings of the IEEE/RSJ International Conference on Intelligent Robots and Systems (IROS), Lausanne, Switzerland, 2002. 2

[29] O. Roesler, A. Aly, T. Taniguchi, and Y. Hayashi. A probabilistic framework for comparing syntactic and semantic grounding of synonyms through crosssituational learning. In Proceedings of the International Workshop on Representing a Complex World: Perception, Inference, and Learning for Joint Semantic, Geometric, and Physical Understanding, in Conjunction with the IEEE International Conference on Robotics and Automation (ICRA), Brisbane, Australia, 2018. 1

[30] A. Rosenberg and J. Hirschberg. V-Measure: A conditional entropy-based external cluster evaluation measure. In Proceedings of the Joint Conference on Empirical Methods in Natural Language Processing and Computational Natural Language Learning (EMNLPCoNLL), pages 410-420, Prague, Czech Republic, 2007. 5

[31] D. Roy, K-Y. Hsiao, and N. Mavridis. Conversational robots: Building blocks for grounding word meanings. In Proceedings of the International Workshop on Learning Word Meaning from Non-Linguistic Data (HLTNAACL), 2003. 1

[32] R. B. Rusu, G. Bradski, and J. Hsu R. Thibaux. Fast 3D recognition and pose using the viewpoint feature histogram. In Proceedings of the IEEE/RSJ International Conference on Intelligent Robots and Systems (IROS), pages 2155-2162, Taipei, Taiwan, 2010. 2

[33] R. Schnabel, R. Wahl, and R. Klein. Efficient RANSAC for point-cloud shape detection. Computer Graphics Forum, 26(2):214-226, 2007. 2

[34] J. Sethuraman. A constructive definition of Dirichlet priors. Statistica Sinica, 4:639-650, 1994. 5

[35] J. M. Siskind. Grounding the lexical semantics of verbs in visual perception using force dynamics and event logic. Journal Of Artificial Intelligence Research (JAIR), 15:31-90, 2001. 1

[36] K. Smith, A. D. M. Smith, and R. A. Blythe. Crosssituational learning: An experimental study of wordlearning mechanisms. Computer Graphics Forum, 35 (3):480-498, 2011. 1, 2, 3, 4

[37] M. Steedman, editor. The Syntactic Process. The MIT Press, Cambridge MA, USA, 2000. 2, 5

[38] M. Steedman and J. Baldridge. Combinatory categorial grammar. In R. Borsley and K. Borjars, editors, NonTransformational Syntax: Formal and Explicit Models of Grammar, pages 181-246. Wiley-Blackwell, 2011. 2

[39] K. Sugiura, N. Iwahashi, H. Kashioka, and S. Nakamura. Learning, generation and recognition of motions by reference-point-dependent probabilistic models. Advanced Robotics (AR), 25:825-848, 2011. 2

[40] M. K. Tanenhaus, M. J. Spivey-Knowlton, K. M. Eberhard, and J. C. Sedivy. Integration of visual and linguistic information in spoken language comprehension. Science, 268(5217):1632-1634, 1995. 1

[41] Y-W. Teh, M. I. Jordan, M. J. Beal, and D. M. Blei. Hierarchical Dirichlet Processes. Journal of the American Statistical Association, 101(476):1566-1581, 2006. 5

[42] S. Tellex, T. Kollar, S. Dickerson, M. R. Walter, A. G. Banerjee, S. Teller, and N. Roy. Approaching the symbol grounding problem with probabilistic graphical models. AI Magazine, 32(4):64-76, 2011. 1

[43] K. Toutanova and M. Johnson. A Bayesian LDA-based model for semi-supervised part-of-speech tagging. In Proceedings of the 20th International Conference on Neural Information Processing Systems (NIPS), pages 1521-1528, Vancouver, Canada, 2007. 3 